\title{
Granular jet impact: probing the ideal fluid description
}

\author{
Patric Müller ${ }^{1} \uparrow$, Arno Formella ${ }^{2}$ and Thorsten Pöschel ${ }^{1}$ \\ ${ }^{1}$ Institute for Multiscale Simulation, Universität Erlangen-Nürnberg, 91052 Erlangen, Germany \\ ${ }^{2}$ Universidad de Vigo, Department of Computer Science, Ourense, Spain
}

(Received 26 September 2013; revised 28 February 2014; accepted 13 April 2014; first published online 24 June 2014)

We investigate the impact of a granular jet on a finite target by means of particle simulations. The resulting hydrodynamic fields are compared with theoretical predictions for the corresponding flow of an incompressible and rotation-free fluid. The degree of coincidence between the field obtained from the discrete granular system and the idealized continuous fluid flow depends on the characteristics of the granular system, such as granularity, packing fraction, inelasticity of collisions, friction and target size. In certain limits we observe a granular-continuum transition under which the geometric and dynamic properties of the particle jet and the fluid jet become almost identical.

Key words: complex fluids, granular media, jets

\section{Introduction}

The impact of a jet of granular material on a fixed target is not only a generic experiment in mechanics, but is also of importance to several industrial and geological applications, such as jet milling (Gommeren et al. 2000), sandblasting (Kuppinger 1990), the formation of craters (Uehara et al. 2003), and several others. Motivated by experiments on water jets (Savart 1833; Clanet 2001), which are well explained by hydrodynamic theory, Cheng et al. (2007) performed experiments on granular jets and found surprising coincidences. In particular, it was found that in both cases the material forms an almost perfect cone after the impact. That is, the material (water or granular) is scattered by a characteristic angle $\psi$, see figure 1(a), with a sharp distribution.

In order to explain the similarity of the granular jet and the liquid jet, Huang, Chan \& Zamankhan (2010), Ellowitz, Guttenberg \& Zhang (2012), Guttenberg (2012) and Sano \& Hayakawa (2012) investigated granular jet impact by means of numerical particle simulations. Such particle simulations provide information about the inner structure of the jet, represented by the hydrodynamic fields deduced from the particle trajectories. This is in contrast to experiments, where it is difficult to obtain the fields of granular systems, except for very low density.

Huang et al. (2010) performed a two-dimensional particle simulation and analysed the pressure field. They identified a stagnation zone in front of the target, where

$\dagger$ Email address for correspondence: patric.mueller@cbi.uni-erlangen.de 
the particles are almost immobile, in contrast to the corresponding fluid flow. The existence of this stagnation zone depends on the surface properties of the particles (Guttenberg 2012). For frictional interaction a stagnation zone is observed, where the absolute velocity of the particles decays exponentially towards the target. For frictionless interaction a stagnation point is observed where the velocity decreases linearly with the distance from this point. Despite these drastic differences of the inner structure, surprisingly, the shape of the jet is almost independent of the frictional properties (Ellowitz et al. 2013). The frictionless case was studied in more detail by Ellowitz et al. (2012), who obtained a striking similarity of the flow field obtained from the particle data to the flow of an ideal incompressible and rotation-free fluid. Picking up on this result, Sano \& Hayakawa (2012) performed three-dimensional simulations of granular jets and attributed the counterintuitive equivalence between the flow of (dense) granular material with finite viscosity and the corresponding flow of an ideal fluid to small shear rates in the granular jet.

The similarity of a scattered granular jet and a jet of a rotation-free incompressible fluid with regard to both their shape and the hydrodynamic fields is far from trivial. In general, granular systems are neither incompressible nor rotation-free. Moreover, the relevance of hydrodynamics to such a system may be questioned at all due to a lack of scale separation (Tan \& Goldhirsch 1998; Goldhirsch 1999, 2003) which is the most fundamental prerequisite of hydrodynamics. Therefore, the question arises of how the granular system converges to its hydrodynamic limit described by ideal fluid flow; that is, how the agreement between ideal fluid flow and particle data depends on the characteristics of the particle systems, such as packing fraction, granularity and dissipative properties.

To this end, we perform an exhaustive parameter study for a quasi-two-dimensional granular jet set-up using highly efficient event-driven molecular dynamics (eMD). As our main result, we will provide conditions under which agreement between the particle system and the corresponding ideal fluid flow may be expected. For non-ideal conditions we quantify the disagreement between the two.

Section 2 describes the set-up of the system and the details of the particle simulation. Subsequently, the results of the particle simulation will be compared with the theory of ideal incompressible rotation-free fluids, applied to the jet problem (for details see $\S 3$ ). In $\S \S 4-6$ we present our main results, namely a comparison of the particle simulations and hydrodynamics. First, we consider the geometry of the problem. In $\S 4$ the scatter angle of the jet is investigated in dependence on the main characteristics of the jet such as granularity, packing fraction, inelasticity, friction and target size. Section 5 is devoted to the contour of the jet. In $\S 6$ we focus on the hydrodynamic fields resulting from hydrodynamic theory and the fields obtained from the particle simulation data by coarse graining. Finally, in $\S 7$ we verify our simulation method by comparing its results with experimental findings.

\section{Particle simulations of the granular jet}

\subsection{System set-up}

We consider a three-dimensional system of identical spherical particles of diameter $d_{p}$, as sketched in figure $1(a)$. By periodically extending the system in the $z$ direction we simulate a quasi-2-D granular jet of width $d_{j}$ impacting a target of width $d_{t}$. We use the 3-D simulation to (a) avoid spurious crystallization which is ubiquitous in two-dimensional systems of identical spheres (see Lubachevsky \& Stillinger 1990; Kapfer et al. 2012), (b) avoid inelastic collapse scenarios which are less probable in 
(a)

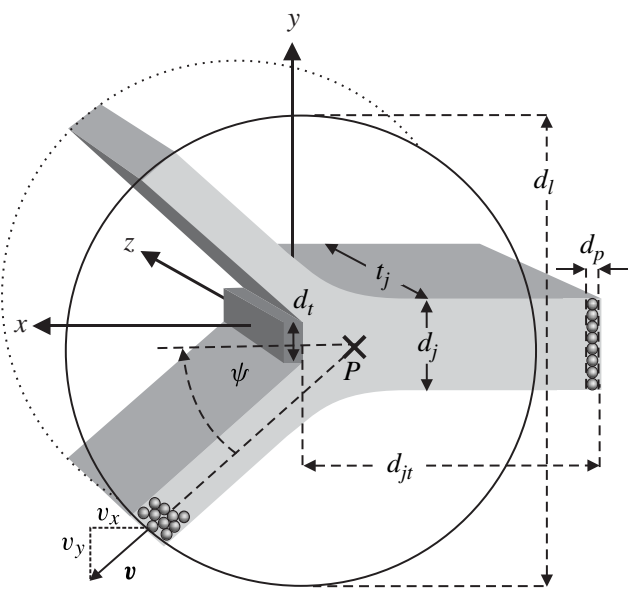

(b)

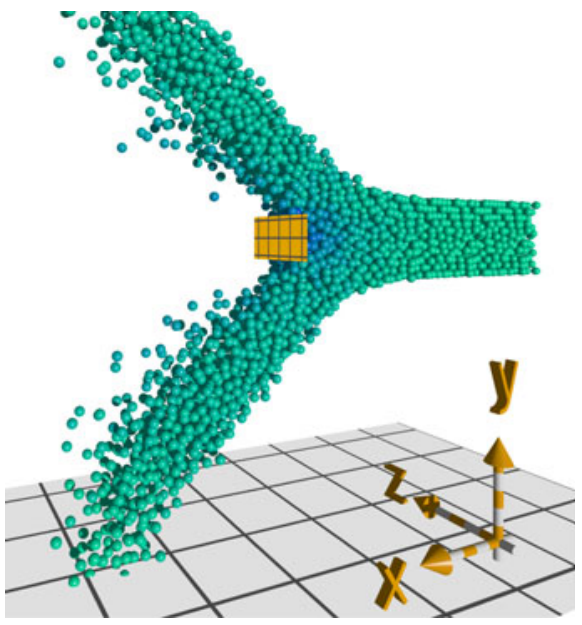

(c)

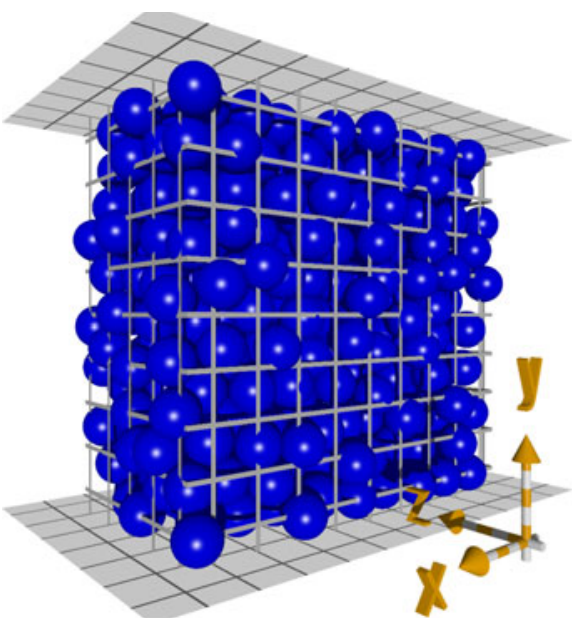

FIGURE 1. (Colour online) (a) Sketch of the jet set-up. (b) Snapshot of the simulation. The grey plane in the lower part as well as the lattice on the target are shown to support the 3-D perspective image. (c) Jet prototype.

3-D than in 2-D (Bernu \& Mazighi 1990; McNamara \& Young 1991) and (c) reduce the fluctuations of the two-dimensional hydrodynamic fields obtained from the particle data.

The particles enter the system through the rectangle

$$
\left(\begin{array}{l}
x \\
y \\
z
\end{array}\right)=\left(\begin{array}{cc}
-d_{j t} \\
-\frac{d_{j}}{2} \cdots & \frac{d_{j}}{2} \\
-\frac{t_{j}}{2} \cdots & \frac{t_{j}}{2}
\end{array}\right) \quad \text { at velocity } \boldsymbol{v}=\left(\begin{array}{c}
v_{x} \\
0 \\
0
\end{array}\right)
$$

forming a stream of rectangular profile parallel to the $x$-axis. The stream is scattered at the rectangular target 


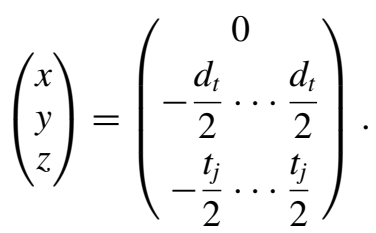

Additionally, the simulation volume is bound by a cylinder of diameter $d_{l}$ the axis of symmetry of which coincides with the $z$-axis. The scatter angle $\psi$ is computed from the coordinates of the particles at the instant when they cross the surface of this cylinder. Velocity fluctuations (often referred to as granular temperature) in the incoming jet are not considered in our investigations. In appendix A we show that a small amount of velocity fluctuation, which is unavoidable in experimental situations, does not invalidate the conclusions drawn in this work.

Initial conditions are generated by means of the algorithm by Lubachevsky \& Stillinger (1990). A rectangular box of size

$$
\left(\begin{array}{l}
x \\
y \\
z
\end{array}\right)=\frac{1}{2}\left(\begin{array}{c}
-l_{j} \ldots l_{j} \\
-d_{j} \ldots d_{j} \\
-t_{j} \ldots t_{j}
\end{array}\right)
$$

with periodic boundary conditions in the $x$ and $z$ directions (transparent lattice in figure $1 c$ ) is filled by $N_{p}$ particles of very small size. Then, a hard-sphere simulation (elastic collisions) is performed during which the radius of the particles is slowly increased. Once the desired packing fraction,

$$
\rho_{j} \equiv \frac{N_{p} \frac{4}{3} R^{3} \pi}{l_{j} d_{j} t_{j}},
$$

is reached, the particle positions and their current radius, $R$, are stored, see figure $1(c)$. This method allows one to produce packings of predefined density, up to random close packing. The particle system produced is used as a prototype for the initialization of the granular jet; that is, it is continuously injected into the simulation volume at $x=$ $-d_{j t}$ at velocities $\boldsymbol{v}=\left(v_{x}, 0,0\right)$ as sketched in figure 1(a). Once the whole prototype is depleted, it restarts seamlessly, which is possible since the prototype is periodic in the $x$ direction.

\subsection{Event-driven dynamics}

We simulate the jet by eMD (see, e.g. Lubachevsky 1991; Pöschel \& Schwager 2005). Within eMD the contact between two particles, using the subscripts $i$ and $j$, is resolved by an instantaneous change of their linear and angular precollisional velocities $\boldsymbol{v}$ and $\omega$ into the postcollisional values

$$
\left.\begin{array}{r}
\boldsymbol{v}_{i}^{\prime}=\boldsymbol{v}_{i}+\frac{m_{e f f}}{m_{i}}\left[\left(1+\varepsilon_{n}\right) \boldsymbol{v}_{n}+\frac{1}{1+\tilde{I}_{e f f}}\left(1-\varepsilon_{t}\right) \boldsymbol{v}_{t}\right], \\
\boldsymbol{v}_{j}^{\prime}=\boldsymbol{v}_{j}-\frac{m_{e f f}}{m_{j}}\left[\left(1+\varepsilon_{n}\right) \boldsymbol{v}_{n}+\frac{1}{1+\tilde{I}_{e f f}}\left(1-\varepsilon_{t}\right) \boldsymbol{v}_{t}\right], \\
\boldsymbol{\omega}_{i}^{\prime}=\boldsymbol{\omega}_{i}+\frac{\tilde{I}_{i}}{R_{i} m_{i}} \frac{m_{e f f}}{1+\tilde{I}_{e f f}}\left(1-\varepsilon_{t}\right) \hat{e}_{r} \times \boldsymbol{v}_{t}, \\
\boldsymbol{\omega}_{j}^{\prime}=\boldsymbol{\omega}_{j}+\frac{\tilde{I}_{j}}{R_{j} m_{j}} \frac{m_{e f f}}{1+\tilde{I}_{e f f}}\left(1-\varepsilon_{t}\right) \hat{e}_{r} \times \boldsymbol{v}_{t},
\end{array}\right\}
$$


where $m_{i}$ and $R_{i}$ stand for the mass and radius of particle $i$ and we use the abbreviations

$$
\begin{gathered}
\hat{e}_{r} \equiv \frac{\boldsymbol{r}_{i}-\boldsymbol{r}_{j}}{\left|\boldsymbol{r}_{i}-\boldsymbol{r}_{j}\right|}, \quad m_{e f f} \equiv \frac{m_{i} m_{j}}{m_{i}+m_{j}}, \\
\boldsymbol{v}_{n} \equiv\left[\left(\boldsymbol{v}_{i}-\boldsymbol{v}_{j}\right) \cdot \hat{e}_{r}\right] \hat{\boldsymbol{e}}_{r}, \quad \boldsymbol{v}_{t} \equiv\left(\boldsymbol{v}_{i}-\boldsymbol{v}_{j}\right)-\boldsymbol{v}_{n}, \\
\tilde{I}_{k} \equiv \frac{m_{k} R_{k}^{2}}{I_{k}}, \quad \tilde{I}_{e f f} \equiv m_{e f f}\left(\frac{\tilde{I}_{i}}{m_{i}}+\frac{\tilde{I}_{j}}{m_{j}}\right), \quad I_{k} \equiv \frac{2}{5} m_{k} R_{k}^{2} .
\end{gathered}
$$

It should be noted that in this work we consider identical particles where $R_{i}=R$ and $m_{i}=m$.

The dissipative properties of particle collisions are characterized by the coefficients of normal and tangential restitution, $\varepsilon_{n}$ and $\varepsilon_{t}$ (see, e.g. Schwager, Becker \& Pöschel 2008; Schwager \& Pöschel 2008), which are assumed constant. In between collision events the particles move along ballistic trajectories. It should be noted that the described method does not resolve permanent contacts.

For dilute systems, eMD can be orders of magnitude faster than traditional force-based MD. For densely packed systems it can be less efficient or even break down completely due to inelastic collapse scenarios where an infinite number of collisions occur in finite time (see, e.g. Luding \& McNamara 1998). As the density in our jet is large, even approaching random close packing, one could hence ask whether eMD is suitable. It turns out that despite the large density of the jet, the vast majority of particles move coherently without much interaction. Velocity fluctuations leading to collisions occur only in a small interaction region in front of the target. While in this particular region eMD is indeed quite inefficient, the overall performance averaged over the whole domain still greatly exceeds that of a similar force-based MD simulation.

\section{Plane steady flow of an ideal incompressible rotation-free fluid}

The velocity field $\boldsymbol{v}(x, y, z)$ of a rotation-free flow may be formally expressed by the velocity potential $\varphi$,

$$
\boldsymbol{v}=\nabla \varphi .
$$

For stationary incompressible flow, the continuity equation takes the form $\nabla \cdot v=0$ and, thus,

$$
\Delta \varphi=0 .
$$

To describe the jet as shown in figure $1(b)$ in the approximation of a stationary incompressible rotation-free flow, (3.2) must be solved for rather complex boundary conditions describing

(a) a source where the material flows in,

(b) two sinks where the material flows out,

(c) a target where the normal component of the velocity vanishes,

(d) free surfaces of yet unknown shape where the pressure is constant, describing the shape of the jet. 
To solve this difficult problem, we apply the technique of conformal maps. An outstanding review of this method may be found in the book by Gurevich (1965); here only the required results are presented.

For plane flow, i.e. $\boldsymbol{v}=\left(v_{x}, v_{y}\right)$, the continuity equation

$$
\boldsymbol{\nabla} \cdot \boldsymbol{v}=\frac{\partial v_{x}}{\partial x}+\frac{\partial v_{y}}{\partial y}=0
$$

suggests that the components of the velocity vector may both be written as derivatives of a certain function $\chi(x, y)$,

$$
v_{x}=\frac{\partial \chi}{\partial y}, \quad v_{y}=-\frac{\partial \chi}{\partial x} .
$$

Using (3.1), this yields

$$
v_{x}=\frac{\partial \varphi}{\partial x}=\frac{\partial \chi}{\partial y}, \quad v_{y}=\frac{\partial \varphi}{\partial y}=-\frac{\partial \chi}{\partial x},
$$

which, in turn, implies that the complex function

$$
\omega \equiv \varphi+\mathrm{i} \chi
$$

of the complex argument $q \equiv x+\mathrm{i} y$ is holomorphic,

$$
\frac{\mathrm{d} \omega}{\mathrm{d} q}=\frac{\partial \varphi}{\partial x}+\mathrm{i} \frac{\partial \chi}{\partial x}=v_{x}-\mathrm{i} v_{y},
$$

where $\omega$ is called the characteristic function or the complex potential and $\mathrm{d} \omega / \mathrm{d} q$ is the complex velocity.

In many cases, solving for $\omega(q)$ is much simpler than solving (3.2) directly. For the case of our problem where the material flows around a finite target, there is another advantage of applying complex algebra. According to the Riemann mapping theorem, under mild preconditions, the flow around any obstacle may be mapped to the known flow around a circular cylinder. If the corresponding map is found, the problem is solved.

For the free jet hitting a fixed finite target (see figure $1 b$ ) this map is known. Furthermore, it is convenient (see Gurevich 1965, Part I, § E) to not consider the problem in the $q=x+\mathrm{i} y$ plane directly but to express the problem using a complex parameter $t$ which, in the case of the jet, varies over the complex unit circle. Using this parameterization, for the jet problem illustrated in figure $1(b)$ the complex potential reads as (Gurevich 1965)

$$
\omega=\frac{s}{\pi}\left[\ln (t-h)+\ln \left(t-\frac{1}{h}\right)-\ln \left(t-\mathrm{e}^{\mathrm{i} \beta}\right)-\ln \left(t-\mathrm{e}^{-\mathrm{i} \beta}\right)\right]
$$

and the transformation back to the original $q=x+$ iy plane is given by

$$
q(t)=\frac{s}{\pi v_{0}} \int \mathrm{d} t \frac{1}{t^{\kappa}}\left[\frac{1}{t-h}+\frac{1}{t-(1 / h)}-\frac{1}{t-\mathrm{e}^{\mathrm{i} \beta}}-\frac{1}{t-\mathrm{e}^{-\mathrm{i} \beta}}\right] .
$$


The parameters $\beta, h$ and $\kappa$ completely determine the exact geometry of the system and $s$ represents the flow rate of the source. For the set-up shown in figure $1(b), \kappa=0.5$ and $h=1$ apply (see Gurevich 1965). The parameter $\beta$ is determined by the scatter angle $\psi$ (see figure $1 a$ ), which, in turn, is related to the ratio $d_{t} / d_{j}$ of the widths of the target and the jet by

$$
\frac{d_{t}}{d_{j}}=\frac{h^{\kappa}}{\pi} \int_{0}^{1} \mathrm{~d} \xi \frac{1}{\xi^{\kappa}}\left[\frac{1}{\xi+h}+\frac{1}{\xi+(1 / h)}-\frac{2(\xi+\cos \beta)}{\xi^{2}+2 \xi \cos \beta+1}\right] .
$$

Consequently, for known $d_{t} / d_{j}$, the parameter $\beta$ or $\psi$, respectively, can be determined by (numerically) inverting (3.10).

To determine the complex potential $\omega$ as a function of our natural coordinates, $x$ and $y$ or $q=x+\mathrm{i} y$, respectively, (3.9) is solved for $t$. (It should be recalled that $\kappa=0.5$ and $h=1$ are determined by the set-up, $s=v_{0} d_{j}$ (Gurevich 1965) and $\beta$ is found from $d_{t} / d_{j}$ by means of (3.10).) For the numerical solution, $v_{0}$ and $d_{j}$ may be chosen as unity without loss of generality since they are the only scales of length and time. The solution $t$ obtained corresponding to the coordinate $q=x+\mathrm{i} y$ is then used in (3.8) to finally compute $\omega(q)=\omega(x, y)$.

If (3.9) does not have a solution $t$, the corresponding complex coordinate $q$ is located outside the jet and $\omega=0$. The components of the velocity field $\boldsymbol{v}(x, y)=\left(v_{x}, v_{y}\right)$ are then directly obtained from (3.5). Apart from this, the assumption of incompressibility and vanishing rotation implies that the flow velocities at upstream and downstream infinity are equal for a free jet. Because incompressible material is considered, conservation of mass further requires that the width of the outgoing jet is half of the width of the ingoing jet.

\section{Granular dynamics versus ideal fluid flow I: scatter angle}

\subsection{Outline}

To compare the properties of the granular jet obtained from particle simulations as described in $\$ 2$ with the corresponding flow of an ideal incompressible and rotationfree fluid, $\S 3$, one can consider the problem on different levels of description. On the lowest level, we look to the only macroscopic, that is collective, characteristic of the problem, which is the scatter angle $\psi$ (see figure $1 a$ ).

At first glance, it is far from obvious that the strongly idealized hydrodynamic modelling is adequate as it is not obvious a priori that the particle jet is incompressible and rotation-free. Moreover, the jet consists of finite-size particles which interact inelastically and frictionally.

Therefore, in this section we discuss the deviation of the scatter angle with regard to all those characteristics of the granular jet that constitute fundamental differences between the description as a particle system and the description as an idealized hydrodynamic system. These are as follows.

(i) The granularity, that is, the ratio of the jet width and the particle diameter, is the main difference between a particle model and a field description.

(ii) The packing fraction is a source of deviations due to the assumption of incompressibility.

(iii) The inelasticity of the particle-particle and particle-target contact establishes a sink of energy which is not included in the hydrodynamic model.

(iv) The friction, that is, the (dissipative) transfer of angular momentum between colliding objects (particles and walls), is not accounted for in the hydrodynamic model. 
In some cases, it is not clear a priori whether a change of one of these characteristics will improve or worsen the agreement between the particle model and the hydrodynamic model. For instance, an increase of the packing fraction reduces the compressibility but also increases the loss of energy due to an enhanced frequency of dissipative collisions.

Finally, in this section we discuss the dependence of the scatter angle on the target size, in comparison with experimental results by Cheng et al. (2007) and simulations by Huang et al. (2010), Guttenberg (2012) and Sano \& Hayakawa (2012). These calculations may also be considered as a pre-exercise for the investigation of large three-dimensional jets in direct comparison with experimental results, see $\$ 7$.

The scatter angle characterizing the jet by a single number is the lowest level of the description. On a higher level, in $\S 5$ we will compare the overall contour of the granular jet as obtained from particle simulations and hydrodynamics. On a yet higher level, in $\$ 6$ we will consider the full hydrodynamic fields as obtained from coarse graining of the particle data of the granular jet and from the hydrodynamic theory described in $\S 3$.

\subsection{Data acquisition and representation}

The particle system was simulated until the jet adopted its stationary shape; the trajectories obtained during this transient were not used for the subsequent analysis. We determined the scatter angle from the trajectories of the particles as follows. For each particle $i$ we computed the velocity $\boldsymbol{v}_{i}=\left(v_{x, i}, v_{y, i}, v_{z, i}\right)$ at the time when the particle leaves the simulation domain (large circle of diameter $d_{l}$ in figure $1 a$ ). The scatter angle $\psi$ is then the average over the individual angles $\psi_{i} \equiv \arctan \left(v_{y, i} / v_{x, i}\right)$. It was checked that the velocity component $\left|v_{z}\right|$ was on average much smaller than the components in the $x$ and $y$ directions. The standard deviation $\sigma_{\psi}$ obtained from the individual angles $\psi_{i}$ indicates the coherence of the granular stream far behind the target; thus, $\sigma_{\psi}=0$ would indicate that all trajectories are perfectly parallel to one another.

In the following $\$ \S 4.4-4.8$ the scatter angle $\psi$ and its standard deviation $\sigma_{\psi}$ are displayed as functions of the characteristic system parameters: granularity, packing fraction, inelasticity, friction and target size. These results obtained from the particle simulation are compared with the results due to the hydrodynamic model developed in $\S 3$ by evaluating (3.10) for given $d_{t} / d_{j}$.

To indicate the applicability of the hydrodynamic theory which relies on incompressible and rotation-free flow, we also plot the vorticity $\langle|\nabla \times v|\rangle$ and the divergence $\langle|\nabla \boldsymbol{v}|\rangle$ averaged over the simulation domain. The method of computing both quantities, divergence and vorticity, will be explained in the context of $\S 6.3$.

\subsection{Generic description of figures 2 and 4-7}

Each of the following $\$ \S 4.4-4.8$ contains a figure that consists of two panels. Panel (b) always displays the mean scatter angle $\psi$ (left ordinate, black line, circles) and its standard deviation, $\sigma_{\psi}$ (right ordinate, green (grey) line, triangles). Panel $(a)$ displays $\langle|\nabla \cdot v|\rangle_{x y}$ (black line, circles) and $\langle|\nabla \times \boldsymbol{v}|\rangle_{x y}$ (green line, triangles), where both quantities are normalized to their maxima individually. The symbols show the data obtained from the particle simulation and the lines are fits to functions specified in the figure captions. The abscissa is the system parameter considered in the current section. The corresponding value of $\psi$ due to the hydrodynamic theory $(\S 3)$ is shown by a dashed line. 


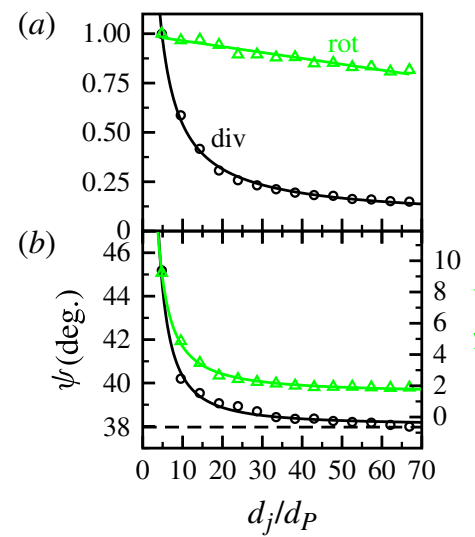

FIGURE 2. (Colour online) The scatter angle $\psi$, its standard deviation $\sigma_{\psi}$ and the average vorticity and divergence of the velocity field as a function of the granularity $d_{j} / d_{p}$. The fit functions are $\psi \approx 38.06+67.46 /\left(d_{j} / d_{p}\right)^{3 / 2}, \sigma_{\psi} \approx 1.53+57.78 /\left(d_{j} / d_{p}\right)^{1.3}, \operatorname{div} \approx 0.05+$ $3.93 /\left(d_{j} / d_{p}\right)^{0.91}$ and $\operatorname{rot} \approx 0.99-0.003\left(d_{j} / d_{p}\right)$. For further details see $\S 4.3$.

If not specified otherwise, the system parameters are $\varepsilon_{n}=0.75, \varepsilon_{t}=1, \rho_{j}=0.6$, $d_{t} / d_{j}=0.5, t_{j} / d_{p}=5, l_{j} / d_{j}=1, d_{j} / d_{p}=40$ and $d_{j t} / d_{j}=4$. Later it will become clear that this choice yields good agreement between the granular dynamics and the ideal fluid flow while keeping the numerical effort during simulation tolerable.

\subsection{Granularity}

The granularity of the system may be quantified as the ratio of the jet width to the particle diameter, $d_{j} / d_{p}$. It should be noted that another meaningful definition of the granularity is given by the ratio of the target width to the particle diameter. Since our set-up is fully described by the given parameters this ratio may be computed. Figure 2 shows the scatter angle $\psi$, its standard deviation $\sigma_{\psi}$ and the average vorticity and divergence of the velocity field.

While the average vorticity decays linearly with $d_{j} / d_{p}$, the average divergence of the flow approaches a small but finite value approximately like $1 /\left(d_{j} / d_{p}\right)$. Consequently, we expect better agreement between the ideal fluid and the granular jet for large $d_{j} / d_{p}$. Indeed, the scatter angle $\psi$ converges to the hydrodynamic value with increasing $d_{j} / d_{p}$ (see figure $2 b$ ).

At first glance, this result seems obvious: the larger the ratio $d_{j} / d_{p}$ is the better the granulate approaches a continuum. On the other hand, figure 3 indicates that the dissipation rate in the material $\left(E_{\text {diss }}\right)$ increases with the grain size $d_{p}$ like $E_{\text {diss }} \propto 1 /\left(d_{p}\right)$. This behaviour may be explained as follows. The dissipation rate is approximately proportional to the frequency of collisions, $v$, and the energy loss per collision, $1-\varepsilon_{n}^{2}$. The collision frequency is given by $v \propto \sqrt{T / m} n d_{p}^{2}$, where $T$ denotes the granular temperature, $m$ is the particle mass and $n$ is the number density of the granulate. As the number density is related to the volume fraction $\rho_{j}$ of the material by $n=$ $\left(6 \rho_{j}\right) /\left(\pi d_{p}^{2}\right)$, we have $v \propto \sqrt{T / m}\left(\rho_{j}\right) /\left(d_{p}\right)$. As $\rho_{j}$ is kept constant when varying the granularity $d_{j} / d_{p}$, this implies $E_{\text {diss }} \propto d_{p}^{-1}$. This in turn contradicts the assumption of an ideal fluid which does not account for dissipation. Consequently, one might also expect that the agreement between particle simulations and hydrodynamics would worsen for large $d_{j} / d_{p}$. This is, however, not observed. 


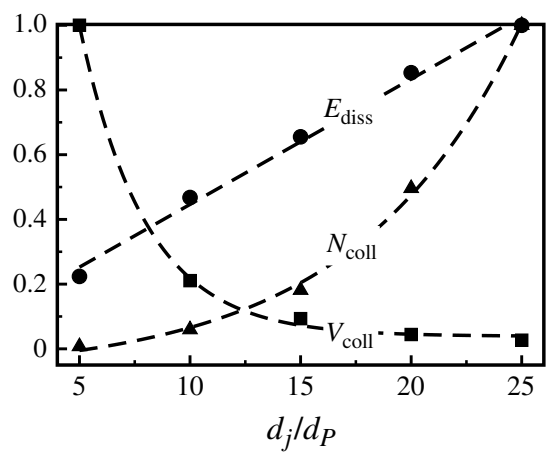

FIgURE 3. The dissipation rate $\left(E_{\text {diss }}, \boldsymbol{\Delta}\right)$ collision rate $\left(N_{\text {coll }}, \mathbf{\square}\right)$ and average impact velocity of two colliding particles $\left(V_{\text {coll }}\right.$, - ) as a function of the granularity $d_{j} / d_{p}$. All values are normalized by the respective maximum value attained in the interval $5<$ $d_{j} / d_{p}<25$. The dashed lines show fits to the data: $E_{\text {diss fit }} \approx 0.1+0.04\left(d_{j} / d_{p}\right), N_{\text {coll }, \text { fit }} \approx$ $-0.08+1.14^{\left(d_{j} / d_{p}-24.4\right)}$ and $V_{\text {coll }, f i t}=0.04+1.4^{\left(4.88-d_{j} / d_{p}\right)}$. The results are obtained from simulation. For further details see $\S 4.3$.

Apart from finding numerical evidence that $\psi\left(d_{j} / d_{p}\right)$ converges to a constant value, it may surprise one that already for $d_{j} / d_{p} \gtrsim 40$ the scatter angle has virtually adopted its asymptotic value, which coincides with the value predicted by hydrodynamic theory. In addition to being of scientific interest, this observation shows that a simulation with $d_{j} / d_{p} \approx 40$ carries already the full information on the physics of the jet formation. Thus, figure 2 suggests that there is no need to simulate jets consisting of particles with diameters more than 40 times smaller than the width of the jet. This, in turn, allows us to limit the number of particles required to obtain reliable results at moderate numerical effort.

\subsection{Packing fraction}

The continuum mechanical theory for the jet described in $\S 3$ assumes incompressible material. For a granular system, the validity of this assumption depends on the packing fraction: a dilute granular gas is much more compressible than a system at random close packing. Therefore, we expect the agreement between the particle system and the ideal fluid to depend on the packing fraction $\rho_{j}$ of the jet, defined by (2.4). Figure 4 displays the corresponding simulation results.

As expected, the divergence characterizing compressibility decreases with the packing fraction while the average curl of the flow follows a parabola with its minimum at $\rho_{j} \approx 0.44$. Therefore, one might expect optimal coincidence between the granular and hydrodynamic systems at $\rho_{j} \gtrsim 0.44$. This is, however, not observed. Instead, $\psi\left(\rho_{j}\right)$ is a parabolic curve with its maximum at $\rho_{j} \approx 0.44$, which is exactly the density where the average curl attains its minimum. The parabola agrees with the hydrodynamic prediction for two values of density, $\rho_{j} \approx 0.26$ and near random close packing, $\rho_{j} \approx 0.62$, corresponding to vanishing compressibility. At the same time, the standard deviation of the scatter angle decreases exponentially with $\rho_{j}$, corresponding to a sharp-edged shape of the granulate behind the target. Although both values, $\rho_{j} \approx 0.26$ and $\rho_{j} \approx 0.62$, correspond to agreement between the angle $\psi$ obtained from the particle simulation and the hydrodynamic theory, only the latter value leads to a jet with a shape that resembles the hydrodynamic prediction, see $\S 5$. 


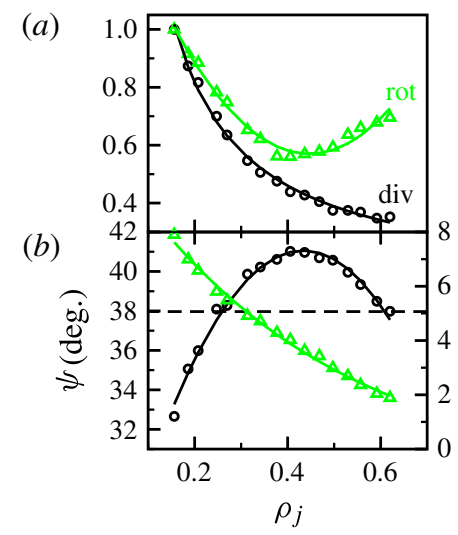

FIGURE 4. (Colour online) Plots of $\psi, \sigma_{\psi}$, div and rot as functions of the packing fraction $\rho_{j}$. The fit functions are $\psi \approx-101.1 \rho_{j}^{2}+87.7 \rho_{j}+22.1, \sigma_{\psi} \approx 1.2+12.5 \exp \left(-2.2 \rho_{j}\right), \operatorname{div} \approx$ $0.11+0.14 / \rho_{j}$ and $\operatorname{rot} \approx 5.2 \rho_{j}^{2}-4.6 \rho_{j}+1.6$. For further details see $\S 4.3$.

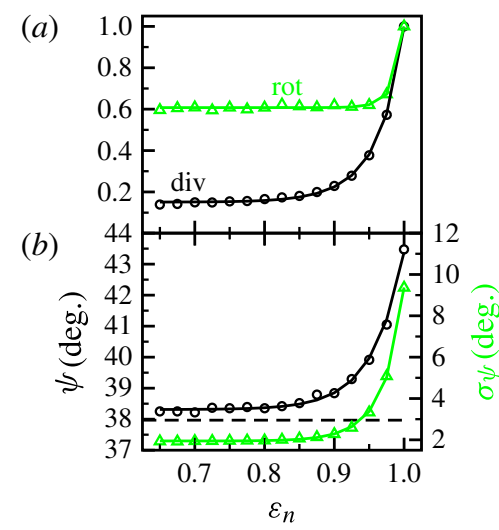

FIGURE 5. (Colour online) The role of inelasticity: $\psi, \sigma_{\psi}$, div and rot as functions of the coefficient of normal restitution $\varepsilon_{n}$. The lines show exponential fits to the data. For further details see $\S 4.3$.

The good agreement for $\psi$ at high packing fraction agrees with our expectation due to the low compressibility of a granulate at random close packing. However, the parabolic shape of $\psi\left(\rho_{j}\right)$ remains unexplained.

\subsection{Inelasticity}

The hydrodynamic theory from $\S 3$ does not consider dissipation; therefore, the question arises of how dissipative particle interaction affects the simulation results. Figure 5 shows the simulation results as a function of the coefficient of normal restitution characterizing the dissipative particle interaction via (2.5).

In contrast to expectations, the scatter angle $\psi$ as well as its standard deviation $\sigma_{\psi}$ increase exponentially with $\varepsilon_{n}$. Agreement between the particle simulation and the hydrodynamic result is obtained for inelastic particles. This observation is accompanied by exponential increase of both the average divergence of the flow and its average vorticity. Thus, the coincidence between the granular jet and the corresponding flow of an ideal incompressible and rotation-free fluid improves with 


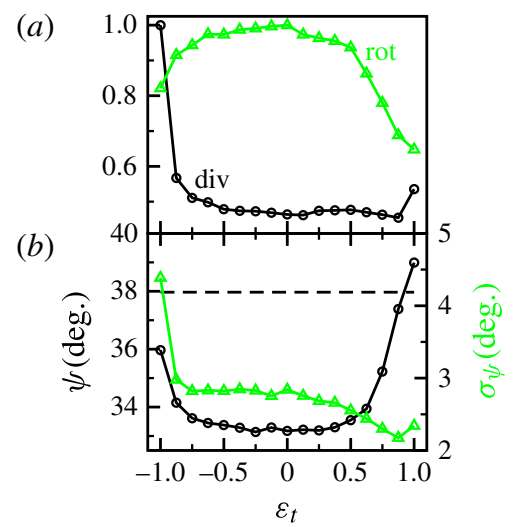

FIgURE 6. (Colour online) The role of friction: $\psi, \sigma_{\psi}$, div and rot as functions of the coefficient of tangential restitution $\varepsilon_{t}$. The lines connect the data points to guide the eye. For further details see $\S 4.3$.

growing inelasticity. The best agreement is reached for $\varepsilon_{n} \lesssim 0.8$; even smaller values of $\varepsilon_{n}$ do not lead to further improvement.

On one hand, this observation may surprise since the hydrodynamic theory does not account for dissipation. On the other hand, the reduction of the relative normal velocity of the particles leads to alignment of the trajectories of adjacent particles, as shown by Brito \& Ernst (1998), which in turn might cause a more coherent particle flow similar to the dynamics of an ideal incompressible and rotation-free fluid.

It should be noted that for $\varepsilon_{n} \lesssim 0.65$ there occur spurious unavoidable inelastic collapse scenarios during the event-driven simulation. This well-known artifact of the hard-sphere model (McNamara \& Young 1991) underlying the event-driven simulation method limits the range of $\varepsilon_{n}$ accessible to event-driven simulations to $0.65 \lesssim \varepsilon_{n} \leqq 1$.

\subsection{Friction}

Within the hard-sphere model, friction is quantified by the coefficient of tangential restitution, $\varepsilon_{t} \in[-1 ; 1]$ (see $(2.5)$ ). This coefficient has two elastic limits. The limit $\varepsilon_{t}=1$ describes perfectly smooth particles such that the rotation cannot be changed by collisions. The other elastic limit, $\varepsilon_{n}=-1$, describes completely elastic reflection of the tangential component of the relative velocity at the contact point, i.e. the particles behave like elastic gearwheels on collision. The inelastic limit, $\varepsilon_{t}=0$, describes a collision where the tangential part of the relative velocity vanishes. Figure 6 shows the simulation data as functions of $\varepsilon_{t}$.

There are two remarkable features: $(a)$ the deviation of the scatter angle from the hydrodynamic prediction follows the vorticity, but not the divergence, and $(b)$ good agreement between hydrodynamics and particle simulation is found for $\varepsilon_{t}$ close to one of its elastic limits. This is qualitatively different from the dependence on $\varepsilon_{n}$ where we find good agreement for the inelastic limit. Out of the elastic limits of $\varepsilon_{t}$ only $\varepsilon_{t} \rightarrow+1$ is of relevance since the other limit, $\varepsilon_{t} \rightarrow-1$, is characterized by a large variance of the scatter angle, that is, incoherent trajectories, which are not observed in the experiment.

\subsection{Target size}

The four parameters discussed in $\$ \S 4.4-4.7$ describe the constitution and material properties of the jet. In the present section, we focus on a geometric property, namely 


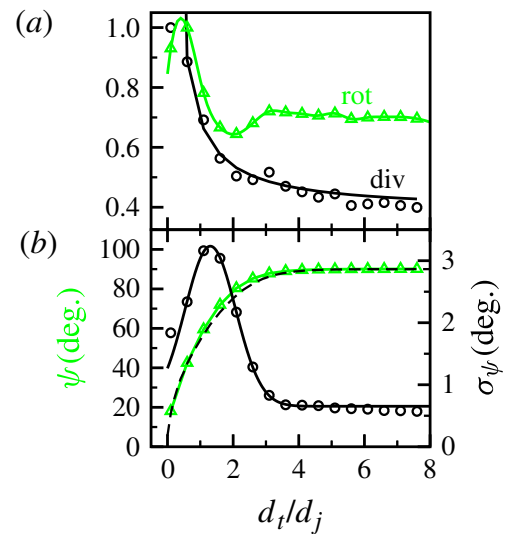

FIGURE 7. (Colour online) Dependence on the target size: $\psi, \sigma_{\psi}$, div and rot as functions of the ratio of target size and jet width, $d_{t} / d_{j}$. The solid black lines show the function $\sigma_{\psi}\left(d_{t} / d_{j}\right)=0.65+2.58 \exp \left(-0.84\left(d_{t} / d_{j}-1.31\right)^{2}\right)(b)$ and $\operatorname{div}=0.39+0.3 /\left(d_{t} / d_{j}\right)(a)$. All other lines are guides to the eye. For further details see $\S 4.3$. It should be noted that in contrast to the previous figures, $\psi$ is plotted by the green line and triangles and $\sigma_{\psi}$ by the black line and circles for better visibility.

the ratio of the target size to the width of the jet, $d_{t} / d_{j}$ (see figure $1 a$ ). All other parameters are maintained at the values specified in $\S 4.3$. These values were chosen such that good agreement was obtained for $d_{t} / d_{j}=0.5$ used in the preceding sections. While the parameters discussed so far, i.e. granularity, packing, inelasticity and friction, do not have counterparts in an idealized hydrodynamic description, the value of $d_{t} / d_{j}$ enters both the hydrodynamic model (via (3.10)) and (obviously) the particle simulation. Figure 7 shows the dependence of the jet properties on the ratio $d_{t} / d_{j}$.

The particle simulation and the corresponding flow of an ideal incompressible and rotation-free fluid are in almost perfect agreement for all target sizes. Only in a narrow interval around $d_{t} / d_{j} \approx 2$ do we see a small deviation, accompanied by some increase of the standard deviation which is, nevertheless, of very small value. While the average divergence of the flow decreases monotonically with $d_{t} / d_{j}$, the average vorticity is almost constant, except for $d_{t} / d_{j} \lesssim 2$. For $d_{t} / d_{j} \gtrsim 4$, the scatter angle approaches its asymptotic limit $\psi \rightarrow 90^{\circ}$.

By varying $d_{t} / d_{j}$ in a wide range while keeping all other parameters invariant, we obtain very good agreement between the results of particle simulation and hydrodynamics. Therefore, we conclude that the good agreement noted in the previous sections is not only a peculiarity of the particular system geometry considered so far but a characteristic feature of the jet set-up.

It should be noted that the dependence of the scatter angle $\psi$ on the ratio of target size to jet width $d_{t} / d_{j}$ was investigated experimentally by Cheng et al. (2007) and in simulations by Guttenberg (2012).

\section{Granular dynamics versus ideal fluid flow II: contour of the jet}

In the previous section we investigated the agreement between the hydrodynamic model of the granular jet and particle simulations for a variety of system and material parameters on the lowest level. Only the scatter angle and its standard deviation were considered; that is, the properties of the jet were condensed to one or two single numbers. For suitable parameters we obtained almost perfect agreement. 


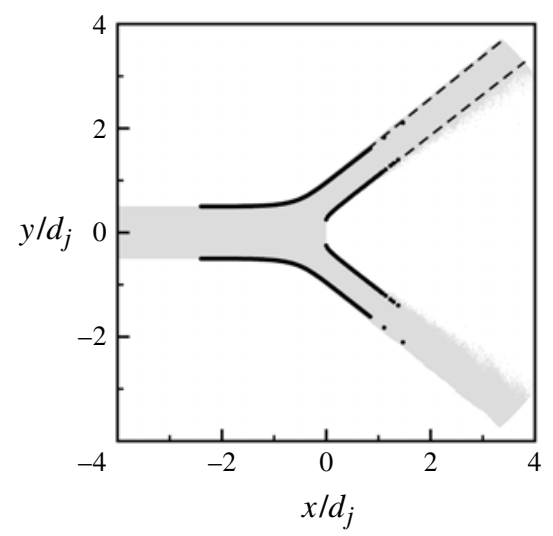

FIGURE 8. The contour of the jet as obtained from the theory of an ideal incompressible and rotation-free fluid (black lines) together with a snapshot of the particle simulation in the stationary state where all particles are shown in grey. For the simulation parameters see $\S 4.3$.

In the current section we check whether this agreement is only for the scatter angle or whether both models describing the jet, granulate and fluid, also agree with regard to the shape (contour) of the jet.

For the ideal incompressible and rotation-free fluid, the shape of the jet may be obtained from the hydrodynamic theory as follows. It is known (Gurevich 1965) that the contour of the jet corresponds to the unit circle in the complex $t$ plane described in $\S 3$. Hence, by solving (3.9) for

$$
t=\mathrm{e}^{\mathrm{i} \theta}, \quad 0 \leqq \theta \leqq 2 \pi,
$$

we obtain a map $\theta \leftrightarrow q$ with $q=x+\mathrm{i} y$ describing the contour of the jet in the $(x, y)$ plane, see figure 8 .

To compare with the simulation results, the hydrodynamic result (black lines in figure 8) was superimposed with a snapshot from the particle simulation where the particles are shown in grey. Up to about one jet width behind the target $\left(x / d_{j} \approx 1\right)$ the shape of the granular jet and the fluid are in almost perfect agreement. For $x / d_{j}>1$, the outer boundaries of the two outgoing branches form a sharp continuous edge, still in perfect agreement with hydrodynamics. The inner surfaces of the outgoing jets appear less coherent; here the particles enter the inner region noticeably, leading to a diffuse structure. This is due to the perturbation the particles experienced through direct contact with the edges of the target. While the outgoing jets as obtained from the hydrodynamic theory are bound by parallel straight lines, this is not the case for the granular flow, which spreads towards the inner region. Apart from these small differences, the agreement between the results of the particle simulation and the corresponding idealized hydrodynamic theory is remarkable, given that there are no adjustable parameters.

\section{Granular dynamics versus ideal fluid flow III: hydrodynamic fields}

\subsection{Outline}

In $\S 4$ we analysed the dependence of the scatter angle as a function of the geometric and material properties of the particle simulation. We demonstrated that for a wide 
range of these parameters, the results of the particle simulation agree very well with the hydrodynamic theory of an ideal incompressible and rotation-free fluid. Assuming a set of parameters out of this range (see $\$ 4.3$ ) the shape of the granular flow agrees almost perfectly with the hydrodynamic theory as well (see $\S 5$ ). Here, assuming the same parameters, we compare the properties of the hydrodynamic results and the particle simulation on a still higher level. To this end, we analyse the inner structure of the flow by examining the hydrodynamic fields.

In the case of the hydrodynamic modelling, the velocity field $\boldsymbol{v}(x, y)$ may be obtained from (3.5). To obtain continuous fields from the discrete particle data of the simulated granular system, we apply the coarse-graining method by Goldenberg \& Goldhirsch (2006), Serero et al. (2008) and Goldhirsch (2010) and some slight generalizations, detailed in the following section.

\subsection{Obtaining hydrodynamic fields from particle data by coarse graining}

We consider a set of particles $i$ of masses $m_{i}$ located at $\boldsymbol{r}_{i}$ and travelling at velocities $\boldsymbol{v}_{i} \equiv \dot{r}_{i}$. The coarse-grained densities of mass and momentum are then defined by

$$
\left.\begin{array}{l}
\rho(\boldsymbol{r}) \equiv \sum_{i} m_{i} \phi\left(\boldsymbol{r}-\boldsymbol{r}_{i}\right) \\
\boldsymbol{p}(\boldsymbol{r}) \equiv \sum_{i} m_{i} \boldsymbol{v}_{i} \phi\left(\boldsymbol{r}-\boldsymbol{r}_{i}\right)
\end{array}\right\}
$$

where $\phi$ is a non-negative scalar function, the spatial integral of which equals unity, with a well-defined width $\omega$ and a single maximum at $\boldsymbol{r}=0$ (see Goldenberg \& Goldhirsch 2006). A good choice is, e.g. a Gaussian

$$
\phi(\boldsymbol{r})=\frac{1}{(\omega \sqrt{\pi})^{3}} \mathrm{e}^{-\left(\frac{r}{\omega}\right)^{2}} .
$$

The coarse-grained velocity field is then defined as

$$
\boldsymbol{V}(\boldsymbol{r}) \equiv \frac{\boldsymbol{p}(\boldsymbol{r})}{\rho(\boldsymbol{r})}
$$

and the fluctuating velocity field of particle $i$ measured at the point $\boldsymbol{r}$ reads as

$$
\boldsymbol{v}_{i}^{f} \equiv \boldsymbol{v}_{i}-\boldsymbol{V}(\boldsymbol{r})
$$

With this, the coarse-grained stress tensor is given by

$$
\sigma_{\alpha \beta}(\boldsymbol{r})=-\frac{1}{2} \sum_{i, j} f_{i j \alpha} r_{i j \beta} \int_{0}^{1} \mathrm{~d} s \phi\left(\boldsymbol{r}-\boldsymbol{r}_{i}+s \boldsymbol{r}_{i j}\right)-\sum_{i} m_{i} v_{i \alpha}^{f}(\boldsymbol{r}) v_{i \beta}^{f}(\boldsymbol{r}) \phi\left(\boldsymbol{r}-\boldsymbol{r}_{i}\right),
$$

where $\boldsymbol{f}_{i j}$ is the force particle $j$ exerts on particle $i$ and $\boldsymbol{r}_{i j} \equiv \boldsymbol{r}_{i}-\boldsymbol{r}_{j}$. The first part of the right-hand side of (6.5) represents the contact stress or collisional stress and the second term the kinetic stress or streaming stress. For coarse-graining scales $\omega$, which are large compared with the separation $\left|\boldsymbol{r}_{i j}\right|$, the integral in (6.5) may be approximated by the coarse-graining function given by Goldenberg \& Goldhirsch (2006),

$$
\int_{0}^{1} \mathrm{~d} s \phi\left(\boldsymbol{r}-\boldsymbol{r}_{i}+s \boldsymbol{r}_{i j}\right)=\phi\left(\boldsymbol{r}-\boldsymbol{r}_{i}\right)+\mathscr{O}\left(\frac{\boldsymbol{r}_{i j}}{\omega}\right) .
$$


The coarse-graining method by Goldenberg \& Goldhirsch (2006) was developed for arbitrary interaction forces $f_{i j}$. For the case of the hard-sphere model, the interaction forces correspond to $\delta$-shaped pulses

$$
f_{i j}(t)=N \delta\left(t-t_{i j}\right)
$$

where $t_{i j}$ is the time of the instantaneous collision between the particles $i$ and $j$. The prefactor $N$ may be obtained by integrating Newton's equation of motion for the collision of two particles,

$$
m_{i} \ddot{\boldsymbol{r}}_{i}=f_{i j}=N_{i} \delta\left(t-t_{i j}\right)
$$

which yields

$$
\boldsymbol{N}_{i}=m_{i}\left(\dot{\boldsymbol{r}}_{i}^{\prime}-\dot{\boldsymbol{r}}_{i}\right)=m_{i}\left(\boldsymbol{v}_{i}^{\prime}-\boldsymbol{v}_{i}\right) .
$$

The collisional part of the stress tensor (first term on the right-hand side of (6.5)) then reads as

$$
\sigma_{\alpha \beta}^{c o l}=-\frac{1}{2} \sum_{t_{i j}=t} m_{i}\left(\dot{\boldsymbol{r}}_{i}^{\prime}-\dot{\boldsymbol{r}}_{i}\right)_{\alpha} r_{i j \beta}\left(t_{i j}\right) \int_{0}^{1} \mathrm{~d} s \phi\left(\boldsymbol{r}-\boldsymbol{r}_{i}+s \boldsymbol{r}_{i j}\right) .
$$

It should be noted that unlike all other coarse-grained quantities considered so far, it is meaningless to evaluate (6.10) at one instant in time, because the probability of encountering an instantaneous collision at exactly one instant in time $\left(t_{i j}=t\right)$ vanishes. Consequently, temporal averages over a certain interval $\left[t_{s} ; t_{e}\right)$ should be considered,

$$
\left\langle\sigma_{\alpha \beta}^{c o l}\right\rangle_{t_{s}}^{t_{e}} \equiv-\frac{1}{2\left(t_{e}-t_{s}\right)} \sum_{t_{i j} \in\left[t_{s} ; t_{e}\right)} m_{i}\left(\dot{\boldsymbol{r}}_{i}^{\prime}-\dot{\boldsymbol{r}}_{i}\right)_{\alpha} r_{i j \beta}\left(t_{i j}\right) \int_{0}^{1} \mathrm{~d} s \phi\left(\boldsymbol{r}-\boldsymbol{r}_{i}+s \boldsymbol{r}_{i j}\right),
$$

where the sum runs over all collisions taking place in the interval $t_{i j} \in\left[t_{s} ; t_{e}\right.$ ).

In the subsequent sections, we present the hydrodynamic coarse-grained fields obtained from the particle trajectories. The results are compared with the fields obtained for the corresponding flow of an ideal incompressible and rotation-free fluid.

\subsection{Divergence and curl}

The two main prerequisites for the hydrodynamic theory described in $\S 3$ are incompressibility and vanishing rotation. Therefore, it is expected that the agreement between this theory and the flow of the granular jet will be best when both the compressibility, measured by the divergence of the flow $\boldsymbol{\nabla} \cdot \boldsymbol{V}$, and the vorticity $\boldsymbol{\nabla} \times \boldsymbol{V}$ are small. Figure 9 displays both quantities, which may be obtained directly from (6.3). The parameters for the granular jet were chosen such that good agreement with the fluid flow is expected due to the analysis of the scatter angle in $\S 4$. Details are given in $\S 4.3$.

Figure 9 may explain the striking agreement between the granular jet and the corresponding hydrodynamic flow described in $\S 3$ by hindsight: in almost the entire simulation domain, both quantities, the divergence and the vorticity, of the granular flow are close to zero. Significant deviations are only encountered in the immediate vicinity of the edges of the target. Additionally, both values are slightly increased along the inner sides of the outgoing jets where the contour of the granular jet also deviates from the hydrodynamics, see figure 8 . 


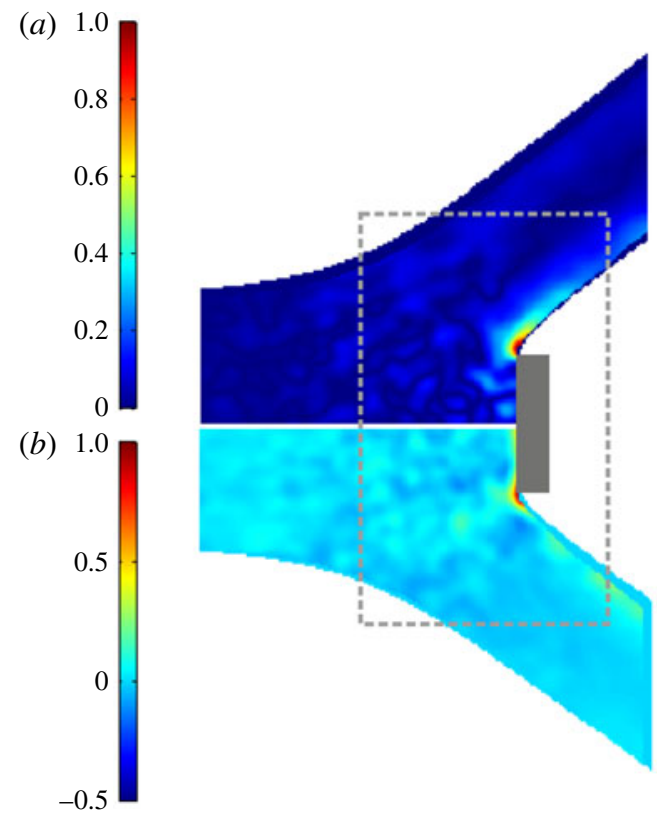

FIGURE 9. (Colour online) Fields of the coarse-grained divergence $\boldsymbol{\nabla} \cdot \boldsymbol{V}(x, y)(b)$ and vorticity $\nabla \times \boldsymbol{V}(x, y)(a)$ in the stationary state. The averaging period was chosen such that the incoming jet travelled a distance of ten particle diameters during the averaging. Both fields are normalized to their maximum values. The grey rectangular area indicates the target. For the parameters see $\S 4.3$. The dashed box indicates the region used to compute the spatial averages $\langle|\nabla \cdot V|\rangle_{x y}$ and $\langle|\nabla \times V|\rangle_{x y}$ shown in figures 2 and 4-7.

The overall vorticity and divergence of the granular system may be quantified by the spatial averages $\langle|\nabla \cdot V|\rangle_{x y}$ and $\langle|\nabla \times V|\rangle_{x y}$ evaluated in the range indicated by the dashed box in figure 9. While the absolute values of these averages are quite meaningless, they may indicate how the degree of fulfillment of the preconditions for the hydrodynamic theory changes on modifying a certain parameter of the particle simulation, such as granularity, packing fraction, etc. Such analyses have been presented in $\$ \S 4.4-4.8$. It should be noted that the choice of the region in which $\langle|\nabla \cdot V|\rangle_{x y}$ and $\langle|\nabla \times \boldsymbol{V}|\rangle_{x y}$ are averaged is basically arbitrary. In appendix B we show that the conclusions drawn in $\$ \S 4.4-4.8$ hold as long as the averaging region is sufficiently large and is chosen such that the incoming jet, the interaction zone in front of the target and the outgoing jets are covered.

\subsection{Absolute velocity}

Let us compare the velocity field $v(x, y)$ for the flow of an ideal incompressible and rotation-free fluid, the main result of $\S 3$, with the velocity field of the granular jet impact obtained from the particle data by coarse graining. Panel $(b)$ of figure 10 shows the field of absolute velocity $|\boldsymbol{v}|(x, y)$ and panel $(a)$ shows the corresponding field for the particle system, obtained from (6.3).

As already discussed by Ellowitz et al. (2012), the overall coincidence between the granular flow and the fluid flow is striking. This is true not only with regard to the geometric shape of the jet, but also with regard to its inner structure. Despite the 


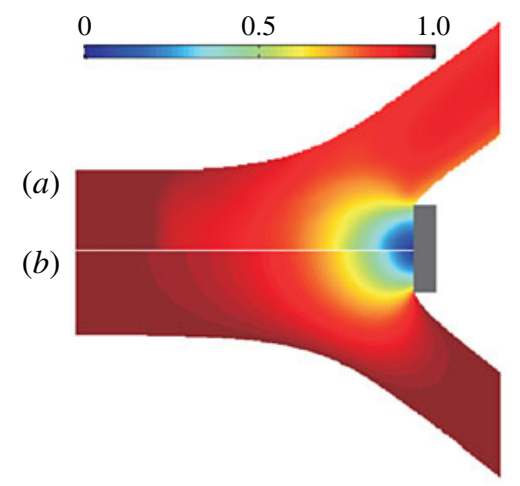

FIgURE 10. (Colour online) The field of the absolute velocity $|\boldsymbol{v}(x, y)|$ for the granular jet due to (6.3) (a) and due to hydrodynamic theory (3.5) (b). The parameters are given in $\S 4.3$.

good agreement, there are two noticeable differences: $(a)$ while the velocities of the ingoing and outgoing jets are identical for the fluid flow, the outgoing velocity of the granular system is smaller than the incoming one; $(b)$ there is a small offset between the absolute velocities of the granular and fluid jets. Both deviations may be attributed to the dissipative nature of particle collisions in the case of the granular flow.

For a more quantitative assessment, in figure 11 the contour lines are shown for some values of the absolute velocity. The figure reveals that the differences in the velocity fields are more significant then expected from figure 10. While right in front of the target, where small absolute velocities are encountered, the contour lines are in very good agreement, for increasing absolute velocity, this coincidence gradually vanishes. For $|\boldsymbol{v}(x, y)| \gtrsim 0.8$ we find large deviations and in the outgoing flow the shape of the contour lines is even of opposite curvature. The astonishing coincidence for small absolute velocities was already highlighted by Ellowitz et al. (2012); however, the substantial differences for larger values of the absolute velocity are not discussed there.

\subsection{Pressure}

The pressure field, $p(x, y)$, of the liquid jet is obtained from the Bernoulli equation valid for incompressible and rotation-free flow,

$$
\rho \frac{|\boldsymbol{v}|^{2}}{2}+p=p_{0},
$$

where the density $\rho(x, y)$ is an arbitrary constant. At the in-flow the pressure vanishes. The constant total pressure $p_{0}$ is, hence, given by

$$
p_{0}=\frac{\rho}{2} v_{x}^{2}
$$

where the corresponding in-flow velocity $v_{x}$ is chosen as unity in the following.

For the granular system, the coarse-grained pressure may be obtained as $1 / 3$ of the trace of the coarse-grained stress-tensor equation (6.5). In analogy to the stress tensor, the pressure is constituted of a kinetic and a collisional part. Both parts are 


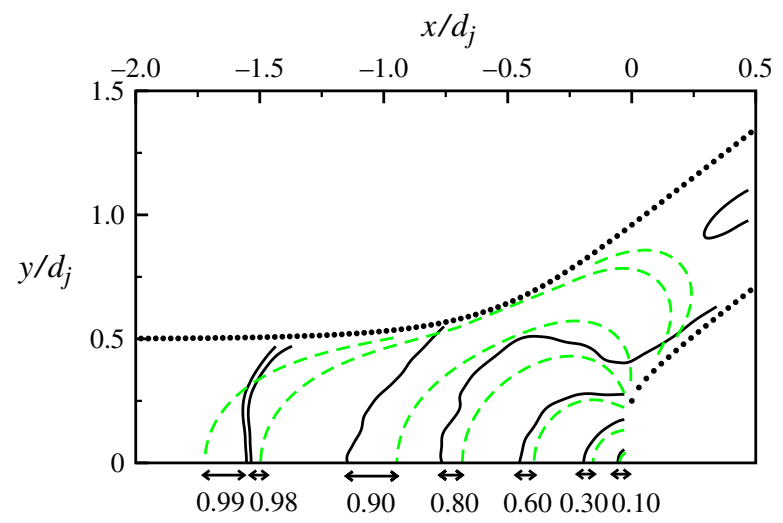

FIGURE 11. (Colour online) Lines of constant absolute velocity for the granular (solid black lines) and the liquid jet (dashed green/grey lines). The dotted line shows the jet contour of the liquid jet as described in $\S 5$. The arrows group the lines for the granular and liquid systems which belong to the same absolute velocity indicated by labels. The parameters are given in $\S 4.3$.

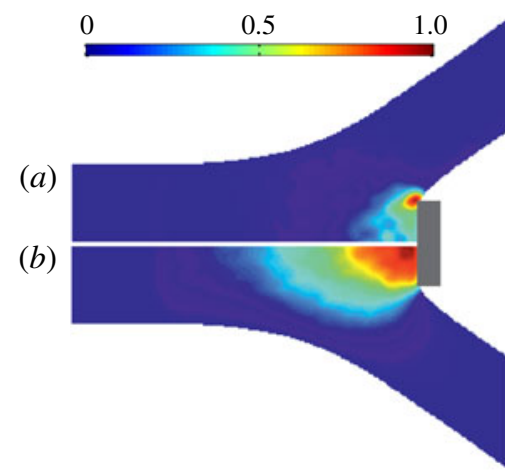

FIgURE 12. (Colour online) Coarse-grained pressure (trace of the stress-tensor equation (6.5)). (a) Kinetic part. (b) Collisional part. The parameters are given in $\S 4.3$.

presented separately in figure 12. (It should be noted that both parts are normalized to their maxima individually. In absolute scale, the kinetic part is approximately 1.5 times larger than the collisional part.) Qualitatively, the collisional part of the granular pressure relates to the field of the absolute velocity in the same way as for the case of the ideal fluid (see (6.12)): the pressure adopts a large value in regions where the absolute velocity is small, and vice versa. The kinetic contribution behaves differently: this part of the pressure is very small in almost the entire domain, except in the vicinity of the edges of the target.

Figure 13 compares the fields of total pressure resulting from the particle simulation of the granular jet with the total pressure obtained from the hydrodynamic theory of an ideal incompressible and rotation-free fluid. Disregarding a small region close to the edge of the target where the kinetic contribution to the pressure dominates (see figure 12), we obtain very good agreement.

For the ideal incompressible and rotation-free fluid, the velocity field is directly related to the pressure field via the Bernoulli equation (6.12). Surprisingly, this relation 


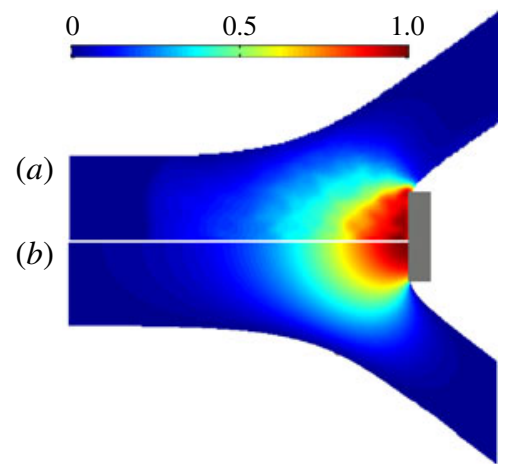

FIgURE 13. (Colour online) The pressure field of the granular flow as obtained by coarse graining of the particle data (trace of (6.5)) (a) and the pressure field of the liquid jet according to $(6.12)(b)$. The parameters are given in $\S 4.3$.

seems to hold to good approximation for the granular flow as well. This is remarkable because in general granular systems are not incompressible and neither may their velocity field be written as the gradient of a velocity potential (see figure 9).

\section{Validation of the hard-sphere modelling}

The hard-sphere model, which was applied to simulate the granular jet, is a strong idealization (see, e.g. Müller, Krengel \& Pöschel 2012; Müller \& Pöschel 2012). Therefore, the question arises whether hard-sphere simulations are capable of describing the physics of real granular jets. To this end, in this section we verify our simulation method by comparing its results with experimental data by Cheng et al. (2007) on the impact of granular jets with circular profiles.

The experiments by Cheng et al. (2007) involve jets with $d_{j} / d_{p} \approx 70$, thus comprising an abundance of approximately 15 million glass or copper beads. Although it is possible to simulate systems containing so many particles using event-driven MD in the dilute state for a reasonable interval of real time on common desktop computers (Bannerman, Sargant \& Lue 2011), for dense systems like the granular jet considered here, eMD becomes less efficient, which limits the feasible number of particles. Fortunately, figure 2 indicates that it is actually not necessary to consider the huge number of particles involved in the experiments. The collective dynamics of the jet does not change significantly when the granularity is changed from $d_{j} / d_{p} \approx 70$ to $d_{j} / d_{p} \approx 40$. Therefore, we compare the experimental results with simulations with approximately four million particles such that $d_{j} / d_{p} \approx 40$.

By applying the above argument three-dimensional simulations approaching the experiment by Cheng et al. (2007) are possible on common desktop computers, where about one week of cpu time is needed to attain a steady-state flow and a good temporal averaging on the steady state. The simulations are analogous to the ones described in $\$ 2$ apart from the shapes of the jet and the target which are now three dimensional, that is cylindrical. Correspondingly, the cylinder bounding the simulation volume (see figure $1 a$ ) is replaced by a sphere and the periodic walls, bounding the simulation in the $z$ direction, are irrelevant.

Figure 14 displays the scatter angle of the circular shaped jet as a function of the ratio $d_{t} / d_{j}$ obtained from the simulation together with the corresponding experimental 


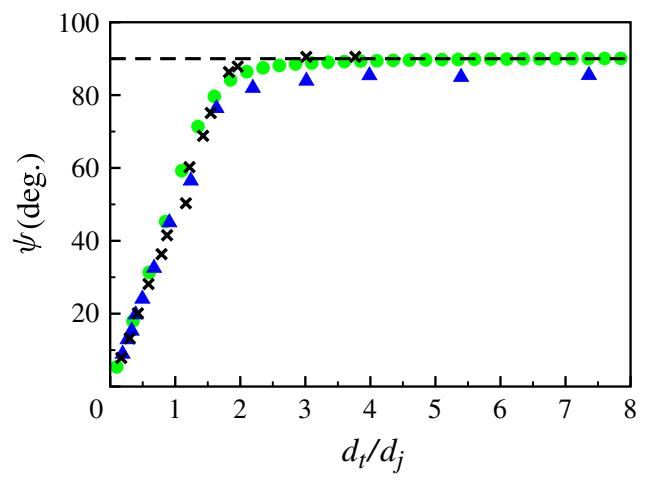

FIgURE 14. (Colour online) The scatter angle $\psi$ as a function of the ratio of the target to the jet diameter $d_{t} / d_{j}$. Black crosses: experimental data by Cheng et al. (2007) for $100 \mu \mathrm{m}$ glass beads; green (grey) circles: three-dimensional hard-sphere simulation; blue (black) triangles: experimental data by Clanet (2001) for a water jet at large Weber number. The dashed line corresponds to $\psi=90^{\circ}$. The parameters of the simulation are $\rho_{j}=0.6$, $\varepsilon_{n}=0.75, \varepsilon_{t}=1, d_{j} / d_{p}=40, l_{j} / d_{j}=1$ and $d_{j t} / d_{j}=8$.

results by Cheng et al. (2007). The simulation results are in perfect agreement with the experimental data for $100 \mu \mathrm{m}$ beads of copper or glass. To highlight the astonishing similarity to fluid flow, figure 14 additionally shows the corresponding experimental data for a water jet in the limit of large Weber number such that surface tension may be neglected (Clanet 2001).

The agreement of the results obtained by simulation and experiment justifies the application of the hard-sphere model and, correspondingly, eMD algorithms a posteriori. It should be noted that the good agreement between simulation and experiment also suggests that interstitial air does not play a role in the experiment as it is not considered by our model.

\section{Summary}

We investigated the impact of a granular jet on an immobile target of finite size by means of event-driven MD exploiting the hard-sphere model for particle collisions. Surprisingly, particular granular jets, characterized by particular parameters, are quantitatively well described by the hydrodynamics of an incompressible and rotation-free fluid, which has been pointed out before by Cheng et al. (2007), Ellowitz et al. (2012), Guttenberg (2012) and Sano \& Hayakawa (2012).

Obviously, this hydrodynamic description of a granular jet is not always (for all sets of parameters) justified since, in general, a granular system is neither continuous nor incompressible nor rotation-free. Therefore, in this paper we raised the question whether there is a hydrodynamic limit for the granular jet such that it can be described by ideal fluid flow and, if it exists, under which conditions this limit is attained.

To this end, we performed an extensive parameter study using event-driven hard-sphere simulations and compared the obtained macroscopic geometric and field characteristics with the corresponding analytical solution of an ideal incompressible and rotation-free fluid.

The most characteristic feature describing the jet impact is the scatter angle, see figure 1(a). Therefore, we investigated how the characteristics of the granular system influence this quantity. These characteristics are the granularity, the packing fraction of 
the impacting jet, the inelasticity of particle collisions, the friction between particles and the target size.

Probably the most obvious difference between a particle description and a hydrodynamic description is the granularity; that is, the ratio between the jet width and the grain diameter $d_{j} / d_{p}$. It was found that the scatter angle of the granular system approaches the value obtained from hydrodynamics like $1 /\left(d_{j} / d_{p}\right)$. Remarkably, the hydrodynamic limit is virtually achieved already for $d_{j} / d_{p} \gtrsim 40$.

Another characteristic feature of a granulate is the dissipative nature of particle collisions. In the hard-sphere approximation, this feature is expressed by the coefficient of normal restitution, $\varepsilon_{n}$. We find that with decreasing $\varepsilon_{n}$, the scatter angle of the granular system converges exponentially to its hydrodynamic limit. For $\varepsilon_{n} \lesssim 0.75$ this limit is practically attained.

It is expected that only densely packed granular jets may be described by the hydrodynamics of an incompressible fluid. To verify this assumption we investigated the scatter angle $\psi$ of the granular system as a function of the packing fraction $\rho_{j}$ of the incoming jet. Surprisingly, there are two values of $\rho_{j}$ where $\psi$ attains its hydrodynamic limit. However, only the higher value of $\rho_{j}$ corresponds to a granular jet with a shape similar to that of the corresponding flow of an incompressible fluid.

Within our simulations, the friction between colliding particles is characterized by the coefficient of tangential restitution $\varepsilon_{t}$. Apparently, friction strongly influences the vorticity of the granular flow. We observed small (average) vorticity for smooth particles where $\varepsilon_{t}=1$. Correspondingly, the scatter angle of the granular jet attains the value predicted for the flow of a rotation-free fluid close to $\varepsilon_{t}=1$. Both $\psi\left(\rho_{j}\right)$ and $\psi\left(\varepsilon_{t}\right)$ approach the hydrodynamic limit $\left(\psi \approx 38^{\circ}\right)$. However, in contrast to $\psi\left(\varepsilon_{n}\right)$ and $\psi\left(d_{j} / d_{p}\right)$ the hydrodynamic limit is approached at finite slope (see figures 6 and 4 ).

While investigating the dependence of the scatter angle on $d_{j} / d_{p}, \varepsilon_{n}, \varepsilon_{t}$ and $\rho_{j}$, the ratio of the diameters of the target and the jet was kept constant. To show that our findings are not just a result of this particular geometry, we showed that the scatter angle of the granular jet is in perfect agreement with that of the fluid flow for all values of $d_{j} / d_{p}$.

After describing the dependence of the scatter angle on the parameters of the granular system, we investigated the details of the hydrodynamic fields. To this end, we considered the set of parameters for which the scatter angle of the granular jet was in optimal agreement with the scatter angle obtained from the hydrodynamic theory of an ideal incompressible and rotation-free fluid.

First, the shape of the jet was considered; that is, the field of density. We found that the outer contour of the granular jet (pointing away from the axis of symmetry) was in perfect agreement with the one of the fluid jet while the inner contour (pointing towards the symmetry axis) exhibited two significant differences. First, the particle flow at the inside was less coherent in comparison with the outer contour. Second, the scatter angle $\psi$ of the particles was slightly smaller than the one predicted by the fluid flow.

The fields of the divergence and the vorticity of the flow obtained from the particle simulation indicated that both quantities are small almost everywhere. Significant differences appeared only at the edge of the target and the inside of the outgoing jets.

Near the target, where small absolute velocities were observed, the contour lines of the field of absolute velocity were in almost perfect coincidence with those of the fluid flow. While this has been observed before by Ellowitz et al. (2012) and Guttenberg (2012), it was found that the contour lines were not comparable at all in regions of high absolute velocities. Even though this was not shown in this paper, a similar 
statement holds for the pressure field of the granular system. Its contour lines compare well with those of the fluid flow in regions where high pressure is observed (near the target). In regions of low pressure the contour lines do not agree at all. Surprisingly, these differences in the fields do not influence the shape of the granular jet which is in almost perfect agreement with the one of the liquid jet everywhere.

The pressure of the granular system decomposes into a kinetic and a collisional contribution. While the collisional part, which dominates almost everywhere, is in perfect agreement with the pressure of the fluid flow, the kinetic part contributes substantially only close to the edges of the target. There, the field of total pressure deviates significantly from the one of the fluid.

Exploiting our findings, we applied event-driven MD to a three-dimensional jet impacting an obstacle. While in three dimensions no analytical result for the corresponding hydrodynamics is available, we could still compare the results of the particle simulation with experimental results. The very good agreement between simulation and experiment justified the hard-sphere model and the event-driven simulation method a posteriori.

\section{Acknowledgements}

We thank Marcus Bannerman, Isaac Goldhirsch, Dan Serero and Nicholas Guttenberg for discussions. The authors gratefully acknowledge the support by the German Science Foundation through the Cluster of Excellence 'Engineering of Advanced Materials'.

\section{Appendix A. The granular temperature of the incoming jet}

The velocity of granular particles separates into a streaming part and fluctuations around the streaming contribution. The fluctuating part is often referred to as the granular temperature. In our simulations we set the granular temperature in the incoming jet to zero. In contrast, a real granulate will always contain some amount of fluctuating velocity as it is difficult to prepare a dense granular jet without generating any velocity fluctuations. In this section we show that small velocity fluctuations, which are unavoidable in experimental situations, may safely be neglected and do not invalidate our conclusions.

Velocity fluctuations of the incoming jet assume their maximum at the outer region of the jet where the particles come into contact with the experimental set-up. Such fluctuations would lead to a widening of the incoming jet. In experiments, e.g. Cheng et al. (2007), the widening angle $\alpha$ (see figure $15 a$ ) is found to be smaller than $1^{\circ}$ (see figure $15 b$ ). From geometry we find $\tan \alpha \approx v_{T} / v_{x}$, where $v_{T}$ may be estimated by the thermal velocity. For $\alpha=1^{\circ}$ this implies $v_{T} \approx 0.02 v_{x}$. In the experiment the velocity fluctuations are initiated when the particles are ejected. From there on, their thermal velocity diminishes due to inelastic collisions. To quantify the impact of temperature on our results, we simulated the corresponding system where the particle velocities at the outlet are distributed according to a Maxwell distribution with the thermal velocity $v_{T} \approx 0.02 v_{x}$. Panel $(c)$ in figure 15 shows the resulting temperature field in the incoming jet. We clearly see that the granular temperature at the particle source $(x=-4)$ rapidly decreases due to collisions. At the position where the jet impacts the target $(x=0)$, the granular temperature is approximately zero. At the boundary of the jet, the granular temperature decreases more slowly than in the interior due to a lack of collision partners. Therefore, the edge of the jet becomes fuzzy and the jet widens by a tiny angle $\lesssim 1^{\circ}$ (see figure $15 c$ ). Panels $(c)$ and $(d)$ of figure 15 hence suggest that the influence of the granular temperature of the incoming jet caused by the interaction of the granulate with the outlet can be neglected. 
(a)

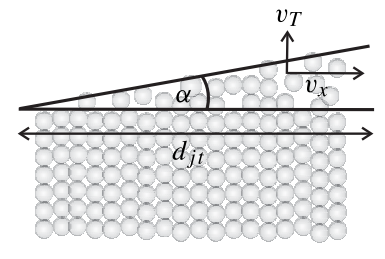

(b)

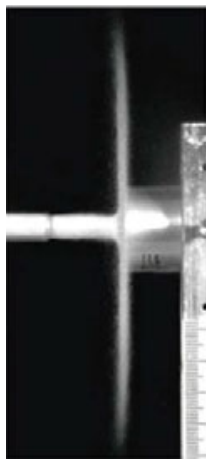

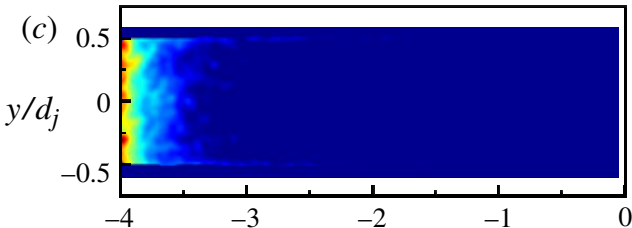

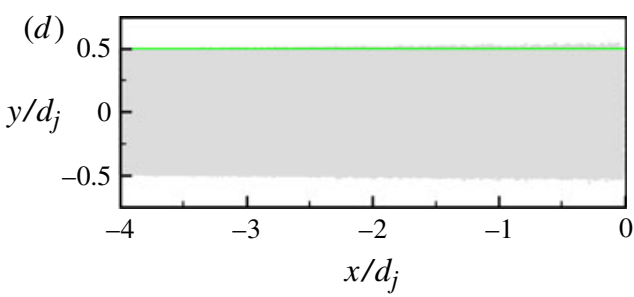

FIgURE 15. (Colour online) (a) The widening of the jet can be used to estimate the thermal velocity $v_{T}$. (b) Experimental realization of the granular jet impact. Reprinted figure with permission from Cheng et al. (2007). Copyright (2007) by the American Physical Society. (c) The temperature field of the incoming jet. (d) The shape of the incoming jet. The deviation of the border of the incoming jet from the straight green (grey) line is due to the initial temperature.

(a)

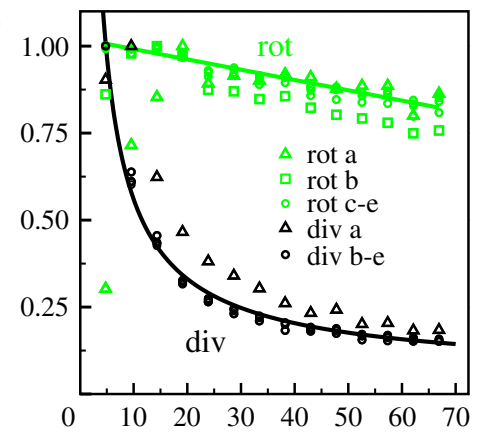

(c)

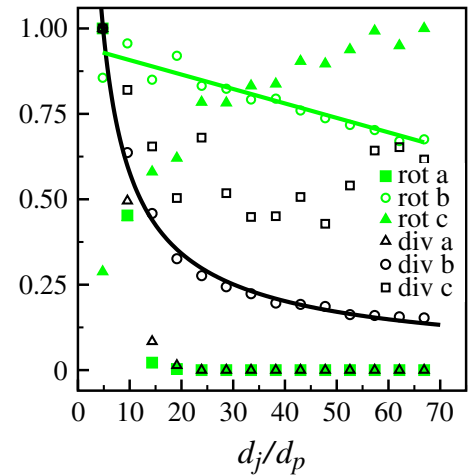

(b)
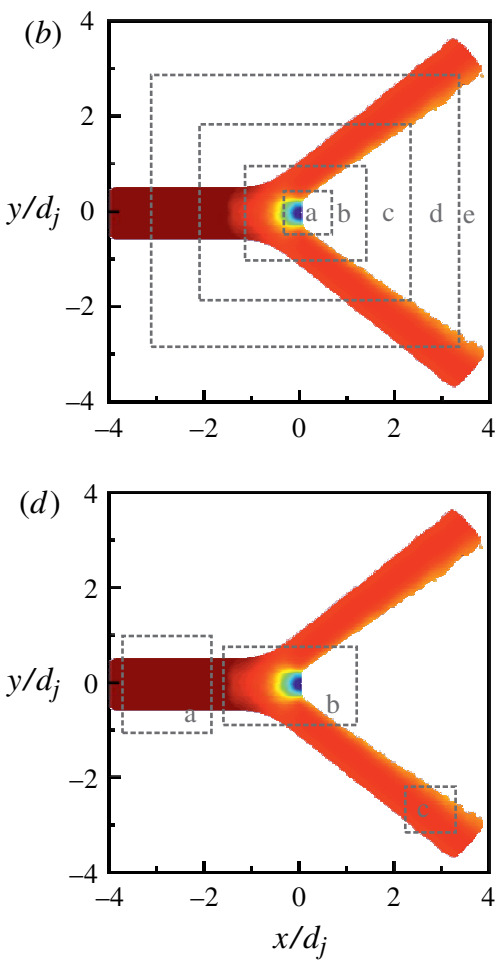

FIgURE 16. (Colour online) (a,c) Average divergence (div, black line/symbols) and rotation (rot, green (grey) line/symbols) of the granular jet flow. The data are the same as shown in figure $2(a, b)$ but for averaging zones of different size and location indicated in $(b, d)$. It should be noted that all sets of data are normalized to their maximum value. 


\section{Appendix B. Average divergence and vorticity}

In $\$ \S 4.4-4.8$ the average divergence and vorticity of the granular flow are used to indicate to what extent the granulate fulfils the prerequisites of the hydrodynamic theory presented in $\S 3$, namely incompressibility and vanishing vorticity. The averages are taken over the region marked in figure 9. To demonstrate that this particular choice is suitable to determine these quantities, we repeat the calculation shown in figure 2(a) but for regions of averaging of different size and location.

Figure 16(a) shows the data of figure 2 where the corresponding regions of averaging are marked in figure $16(b)$. When computing the average divergence, regions b-e all yield the same result (curves div b-e); only div a corresponding to region a deviates noticeably. For the average rotation, averaging over regions c-e yields almost identical results, whereas averaging over the smaller regions a or $b$ leads to deviations. However, not only the size of the averaging zone matters, but also its location. Figure $16(c, d)$ compares the results when averaging over three zones, $\mathrm{a}, \mathrm{b}$ and $\mathrm{c}$, of different location. Here, only averaging over zone b yields results in agreement with figure 2 ; the other zones are not representative for the vorticity and divergence of the jet problem.

From the results shown in figure 16 we conclude that the results presented in $\S \S 4.4-4.8$ are relatively insensitive with respect to the choice of the averaging zone, provided that it covers the interaction zone in front of the target, including the incoming jet and the outgoing jets.

\section{REFERENCES}

Bannerman, M. N., Sargant, R. \& Lue, L. 2011 DynamO: a free $\mathrm{O}(\mathrm{N})$ general event-driven simulator. J. Comput. Chem. 32, 3329-3338.

BERnU, B. \& MAZIGHI, R. 1990 One-dimensional bounce of inelastically colliding marbles on a wall. J. Phys. A 23, 5745-5754.

BRito, R. \& ERnst, M. 1998 Extension of Haff's cooling law in granular flows. Europhys. Lett. 43, 497-502.

Cheng, X., Varas, G., Citron, D., Jaeger, H. M. \& Nagel, S. R. 2007 Collective behavior in a granular jet: emergence of a liquid with zero surface tension. Phys. Rev. Lett. 99, 188001.

Clanet, C. 2001 Dynamics and stability of water bells. J. Fluid Mech. 430, 111-147.

Ellowitz, J., Guttenberg, N. \& Zhang, W. W. 2012 Perfect fluid flow from granular jet impact. arXiv:1201.5562v1 [physics.flu-dyn].

Ellowitz, J., Turlier, H., Gutenberg, N., Zhang, W. W. \& Nagel, S. R. 2013 Still water: dead zones and collimated ejecta from the impact of granular jets. Phys. Rev. Lett. 111, 168001.

GoldenberG, C. \& Goldhirsch, I. 2006 Continuum mechanics for small systems and fine resolutions. In Handbook of Theoretical and Computational Nanotechnology (ed. M. Rieth \& W. Schommers), vol. 4, pp. 329-386. American Scientific.

GoldhiRSCH, I. 1999 Scales and kinetics of granular flows. Chaos 9, 659-672.

Goldhirsch, I. 2003 Rapid granular flows. Annu. Rev. Fluid Mech. 35, 267-293.

GOLDHIRSCH, I. 2010 Stress, stress asymmetry and couple stress: from discrete particles to contiuous fields. Granul. Matt. 12, 239-252.

Gommeren, H. J. C., Heitzmann, D. A., Moolenaar, J. A. C. \& Scarlett, B. 2000 Modelling and control of a jet mill plant. Powder Technol. 108, 147-154.

Gurevich, M. I. 1965 Theory of Jets in Ideal Fluids. Academic Press.

Guttenberg, N. 2012 Microscopic dissipation in a cohesionless granular jet impact. Phys. Rev. E 85, 051303.

Huang, Y. J., Chan, C. K. \& Zamankhan, P. 2010 Granular jet impingement on a fixed target. Phys. Rev. E 82, 031307. 
Kapfer, S. C., Mickel, W., Mecke, K. \& Schröder-Turk, G. E. 2012 Jammed spheres: Minkowski tensors reveal onset of local crystallinity. Phys. Rev. E 85, 030301.

KUPPinger, G. 1990 Strahlen von Oberflächen. Metall 44, 43-52.

Lubachevsky, B. D. 1991 How to simulate billiards and similar systems. J. Comput. Phys. 94, 255-283.

Lubachevsky, B. D. \& Stillinger, F. H. 1990 Geometric properties of random disk packings. J. Stat. Phys. 60, 561-583.

Luding, S. \& MCNAMARA, S. 1998 How to handle the inelastic collapse of a dissipative hard-sphere gas with the TC model. Granul. Matt. 1, 113-128.

MCNAmara, S. \& Young, W. R. 1991 Inelastic collapse and clumping in a one-dimensional granular medium. Phys. Fluids 4, 496-504.

Müller, P., Krengel, D. \& Pöschel, T. 2012 Negative coefficient of normal restitution. Phys. Rev. E 85, 041306.

MÜlleR, P. \& Pöschel, T. 2012 Oblique impact of frictionless spheres: on the limitations of hard sphere models for granular dynamics. Granul. Matt. 14, 115-120.

Pöschel, T. \& Schwager, T. 2005 Computational Granular Dynamics - Models and Algorithms. Springer.

SANO, T. G. \& HAYAKawA, H. 2012 Simulation of granular jets: is granular flow really a perfect fluid? Phys. Rev. Lett. 86, 041308.

SAVART, F. 1833 Mémoire sur le choc d'une veine liquid lancée contre un plan circulaire. Ann. Chim. Phys. 54, 55-87.

Schwager, T., Becker, V. \& Pöschel, T. 2008 Coefficient of tangential restitution for viscoelastic spheres. Eur. Phys. J. E 27, 107-114.

SCHWAGer, T. \& PösChel, T. 2008 Coefficient of restitution for viscoelastic spheres: the effect of delayed recovery. Phys. Rev. E 78, 051304.

Serero, D., Goldenberg, C., Noskowicz, S. H. \& Goldhirsch, I. 2008 The classical granular temperature and slightly beyond. Powder Technol. 182, 257-271.

TAn, M. -L. \& Goldhirsch, I. 1998 Rapid granular flows as mesoscopic systems. Phys. Rev. Lett. 81, 3022-3025.

Uehara, J. S., Ambroso, M. A., Ojha, R. P. \& Durian, D. J. 2003 Low-speed impact craters in loose granular media. Phys. Rev. Lett. 90, 194301. 